\title{
Los sistemas de investigación de la muerte innatural: coroners y medical examiners
}

\section{Unnatural death investigation systems: coroners and medical examiners}

\section{JD. Sánchez Pérez}

Doctor en Medicina. Médico Forense. Jefe de Servicio de Laboratorio Forense.

Correspondencia: José Domingo Sánchez Pérez Instituto de Medicina Legal de Palencia, Salamanca y Valladolid. Paseo Arco de Ladrillo, 72-74. 47007 Valladolid E-mail: jd.sanchez@justicia.es

Fecha de recepción: 12. NOV. 2012

Fecha de aceptación: 01. JUL. 2013

\section{Resumen}

La muerte innatural es investigada por las autoridades de una forma especial debido a razones legales. Hay dos modelos principales para este propósito; uno está basado en la tradición de la Common Law, el sistema coroner, y otro en la investigación del delito. El artículo entra en detalles del sistema coroner y de su trasformación, el sistema medical examiner. Finalmente, se hacen varias consideraciones sobre la investigación de la muerte en España.

Palabras clave: Coroners y Medical Examiners. Legislación y jurisprudencia. Medicina Forense.

\section{Abstract}

Unnatural death is investigated by authorities in a special way because of legal reasons. There are two main models to this purpose; the one is based in Common Law tradition, the coroner system, and the other in the crime investigation. The paper goes into details of the coroner system and also of its transformation, the medical examiner system. Finally, several considerations are made about death investigation in Spain.

Key words: Coroners and Medical Examiners. Legislation \& jurisprudence. Forensic Medicine.

\section{Introducción}

La muerte innatural es toda la que no es natural. En España, es la que tiene las causas definidas por el artículo 340 de la Ley de Enjuiciamiento Criminal, que son la violenta o la sospechosa de criminalidad, siendo esta última una categoría provisional para las muertes aparentemente naturales cuyas circunstancias no son claras. Se trata, por tanto, de un concepto jurídico y no médico, tal vez también de naturaleza médico legal. La característica principal y definitoria, patognomónica que se diría en Medicina, de la muerte innatural es que debe ser investigada por las autoridades de una forma especial, fuera de lo ordinario. Esta indagación diferente debe hacerse por razones que son legales y no médicas, no son las mismas por las que las autopsias clínicas se hacen en muertes naturales. La muerte innatural es aquella causa y circunstancias que deben ser averiguadas específicamente porque la ley lo dice así. Además, es una categoría de carácter internacional, aunque las causas que se consideran no naturales y la manera de investigar estas muertes varían según los países y su tradición jurídica.

En realidad, todas las muertes son investigadas, ya que la emisión de un certificado de defunción en las muertes naturales no deja de ser una obligación legal y el diagnóstico en el contenido no es sino una opinión basada en el estudio del caso. Pero en el caso de las muertes naturales se sigue un trámite administrativo que podría llamarse ordinario, mientras que a las muertes innaturales se les aplica un tratamiento extraordinario y diferente.

Existen dos modelos principales de investigación de la muerte innatural. El primero de ellos es común en la Europa continental y se caracteriza porque la investigación de las muertes está subordinada a la del delito; las distintas formas de este modelo son cono- 
cidas como "sistemas civiles". El segundo de ellos es predominante en los países de cultura anglosajona y está basado en la tradición de la Common Law, que se caracteriza por una investigación autónoma de las muertes. Es conocido como sistema Common Law y también, por contraposición al anterior, las variedades de este modelo podrían denominarse "sistemas autónomos". La mayoría de los países con la tradición de la Common Law tienen un sistema coroner o algo que se le parece, que investiga la mayoría (o la totalidad) de tipos de muerte innatural, incluso si no implica delito. Sin embargo, esto no es así con los sistemas civiles, en los que las pesquisas sobre las muertes son llevadas a cabo (en todo caso) mayoritariamente como una parte de la investigación criminal. También se diferencia en que la pesquisa del coroner es, en principio, un acontecimiento público, en el que la evidencia se presenta ante un tribunal público, mientras que como norma en los sistemas basados en la ley civil la investigación es confidencial, aunque las conclusiones pueden ser públicas ${ }^{1}$.

En cada uno de estos modelos, el autónomo y el civil, pueden distinguirse a su vez dos variedades. En el caso del modelo Common Law existen los sistemas coroner, por mantener esta figura, y los sistemas medical examiner, que representan una evolución de los anteriores. En el caso de los sistemas civiles, la función de investigar la muerte, es decir, de instruir el procedimiento, puede ser del juez o del fiscal. Dado que por nuestra cultura jurídica estamos mucho más familiarizados con los sistemas civiles que con los autónomos, pasaremos a considerar extensamente sólo éstos, para finalizar con unas consideraciones sobre la investigación de las muertes innaturales en España.

\section{El sistema coroner}

Un coroner es un empleado público cuya función es la de investigar las muertes que ocurren en circunstancias excepcionales. De acuerdo con el informe presentado en el Parlamento del Reino Unido en el año 2003, relativo a la certificación e investigación de la muerte en Inglaterra, Gales e Irlanda del Norte, las características del sistema coroner son dos$^{2}$ :

- Es un servicio de investigación especializada centrado solamente en la investigación de las muertes.

- La búsqueda de la causa médica o violenta de la muerte de un individuo toma la forma de una investigación con estilo judicial de sus circunstancias.

\section{Historia}

Según el Profesor Knight ${ }^{3}$, se trata de una institución original inglesa, aunque debería decirse mejor normanda, cuya historia se remonta a la Edad Media. El rey Ricardo Corazón de León se encontraba en una situación de desesperada necesidad de dinero, no en pequeña proporción por el enorme rescate del secuestro al que le sometió Leopoldo de Austria al volver de las cruzadas. Unos años antes una comisión real había destituido por corruptos a todos los representantes reales en los condados (los sheriff, palabra derivada de "shire-reeve"). Hubert Walter, Arzobispo de Canterbury, verdadero gobernante del reino por las prolongadas ausencias del monarca, decidió que cada condado eligiera a tres caballeros y un clérigo para guardar los alegatos de la corona (custos placitorum coronæ) hasta la llegada de la corte de justicia itinerante (General Eyre) encargada de imponer las penas. Los circuitos de esta corte duraban siete o más años, por lo que, a menos que se documentaran los hechos de forma conveniente, por la tardanza podrían llegar a perderse importantes ingresos para la corona en forma de multas o fianzas. Estos escritos se registraban en pergaminos conocidos como los Coroners' Rolls, muchos de los cuales han sobrevivido hasta hoy.

La labor más importante de estos representantes de la corona era la de investigar las muertes rápidas, no sólo asesinatos y homicidios, sino también las accidentales y naturales, sin olvidar los suicidios. Sin embargo, su objetivo esencial no era descubrir al autor, sino dejar constancia escrita de todas las circunstancias y todos los implicados de una u otra manera (y de sus propiedades) a la espera de la llegada del tribunal itinerante, el cual no era raro que impusiera cuantiosas multas (al arbitrio del tribunal, por lo que se denominaban amercements) por cualquier desviación del complejo procedimiento prescrito ante el hallazgo de un cadáver. Por ejemplo, el municipio que no avisara al coroner de este hallazgo cometía un delito que podía ser fuertemente multado, lo mismo que enterrar el cuerpo antes de la llegada del coroner. En la práctica, no era infrecuente ignorar la presencia de un cadáver o hacerlo desaparecer discretamente bajo tierra o, incluso, en el pueblo de al lado, para que éste "cargase con el muerto".

Una de las primeras tareas de la investigación de la muerte era la de averiguar la identidad del fallecido, ya que si era normando se imponía una fuerte multa a la comunidad, conocida como murdrum. Ocurrida en 1066 la conquista normanda, los invasores impusieron que cualquiera que apareciera asesinado se presumía normando, por lo que sólo podía evitarse la 
multa mediante la prueba (Presentment of Englishry) de que el fallecido era sajón. Además, un fallecimiento confería al coroner el poder de confiscar o valorar para la corona cualquier objeto que hubiera causado la muerte (el llamado deodand, del latín "Deo dandum") no importaba el tamaño o la naturaleza; en el siglo XIX, cuando se abolió esta práctica, Io habían sido objetos tan grandes como locomotoras y hasta un barco. Cabe destacar que los suicidios se investigaban porque eran considerados un crimen y todos sus bienes muebles e inmuebles, incluidas sus tierras, no iban a parar a sus herederos, sino al rey ${ }^{4,5}$.

Las funciones del coroner fueron variando con el tiempo. Entre las funciones que han desaparecido están las derivadas del derecho de santuario, de los naufragios, de las capturas de ballenas y esturiones (considerados como de propiedad real) y ciertas apelaciones, declaraciones y abjuraciones. A finales del siglo XV, los poderes fiscales que poseían fueron asumidos por los jueces de paz. En 1836, a resultas de la preocupación por los homicidios encubiertos resultante de las atrocidades de Burke y Hare, los conocidos homicidas que vendían los cuerpos de los que asesinaban a los estudiosos de la anatomía, se promulgaron leyes bajo las cuales no podía haber ninguna inhumación sin un certificado del Registro de Nacimientos y Muertes o una orden del coroner, así como se daban poderes al coroner para requerir la ayuda de un médico y para ordenar la necropsia. En 1926 se dispuso que los coroners aplazaran la investigación si una persona era acusada del asesinato $u$ homicidio del fallecido y en 1977 se excluyeron las cuestiones relativas a la responsabilidad criminal del objeto de las investigaciones del coroner ${ }^{6}$. Como puede verse, el coroner en Inglaterra fue evolucionado desde la figura de un recaudador de impuestos medieval hasta la actual de un funcionario judicial encargado de la investigación de las muertes súbitas, violentas o no naturales ${ }^{4}$. Una función que no obstante se mantiene en la actualidad es la referida al hallazgo de tesoros, ya que se considera que pertenecen a la corona: el hallazgo de cualquier objeto que pueda ser considerado legalmente "tesoro" debe ser comunicado al coroner, bajo pena de cárcel y multa, el cual investigará si lo es o no; en caso positivo, se ofrece su adquisición a los museos nacionales; no obstante, el que encuentra el tesoro, tiene derecho a que se le recompense con su precio ${ }^{7}$.

\section{Funciones}

La figura del coroner para el estudio de los fallecimientos sospechosos existe en Inglaterra y Gales; también en Irlanda del Norte y en la República de
Irlanda; sin embargo, no la hay en Escocia. También se ha extendido a los países con lazos históricos con el Reino Unido: Canadá, Australia, Nueva Zelanda, Hong Kong y Kenia, entre otros. Existe asimismo en parte de los Estados Unidos, donde en algunos Estados ha evolucionado, como luego veremos, hasta exigirse una titulación médica para su desempeño, convirtiéndose en los denominados medical examiners.

En Inglaterra y Gales ${ }^{8-10}$, son los titulares de un cargo judicial independiente, nombrado y pagado por las autoridades locales. Hay tres clases de coroners: coroners ex officio, son los jueces de la High Court, quienes pueden ejercer las funciones de un coroner; coroners de distrito, son los nombrados para cada una de las divisiones del territorio (jurisdicciones); y coroners específicos como el de la Casa Real. Para ser un coroner desde 1926 se requiere ser abogado o médico (a veces es ambas cosas) y haber estado en activo durante al menos cinco años. Cada coroner tiene un sustituto, de manera que siempre hay uno disponible a cualquier hora. Cuentan con personal auxiliar, los coroner's officers, tanto para los trámites burocráticos como para las investigaciones; en algunos sitios trabajan a tiempo completo y en otros son policías que lo hacen a tiempo parcial para la oficina del coroner.

Está establecido que se debe dar parte al coroner de los fallecimientos que se producen en las siguientes circunstancias:

- Ningún médico atendió al fallecido en su última enfermedad.

- Aunque fuera atendido por el médico en su última enfermedad, no fue atendido en los catorce últimos días ni tras su muerte.

- La causa de muerte parece ser desconocida.

- La muerte ocurrió durante una intervención quirúrgica o antes de la recuperación de los efectos de la anestesia.

- La muerte fue debida a un accidente, enfermedad o intoxicación industriales.

- La muerte fue súbita o inesperada.

- La muerte fue no natural.

- La muerte fue debida a violencia o negligencia.

- La muerte fue en otras circunstancias sospechosas.

- La muerte ocurrió en prisión, en custodia policial u otra forma pública de detención.

La competencia del coroner sobre un caso está determinada por el lugar donde se halle el cuerpo y no por 
aquel donde ocurrió la muerte. Una vez notificado el fallecimiento al coroner, éste decide investigarlo o no; en caso afirmativo, no puede inscribirse en el registro hasta que sus investigaciones finalicen. El coroner tiene derecho a obtener copias de la información médica que sea relevante y necesaria para sus investigaciones. Algunas veces el médico puede discutir el caso con el coroner y éste puede decidir que no son necesarias más investigaciones, ya que la muerte es debida a causas naturales, y comunica al registro que puede admitir el correspondiente certificado de defunción que emita el médico. Por ejemplo, en los casos en los que se da parte de una muerte por no haber sido asistida la persona por el médico en los catorce días previos, el coroner consulta con el médico del fallecido, y normalmente no se precisa la autopsia. En otras ocasiones, el coroner ordenará la práctica de la autopsia por un patólogo de su elección. Los allegados son notificados de ello, aunque no pueden oponerse a su práctica; sí que pueden dar cuenta al coroner de sus dudas o preocupaciones al respecto y tienen derecho a nombrar a un médico de su elección para que les represente en dicho examen, así como a obtener una copia del informe de autopsia cuando se emita. Si la post-mortem, término coloquial que se emplea con frecuencia en Inglaterra para referirse a la autopsia, demuestra que la causa fue natural, el coroner emitirá una notificación sobre este extremo para que la muerte pueda ser inscrita en el registro. Esta notificación se suele remitir directamente al registro, aunque en algunos casos se entrega a los familiares para su tramitación.

\section{La encuesta}

Si la causa de la muerte sigue siendo desconocida tras la operación autópsica y las pruebas complementarias, o el coroner tiene razones para sospechar una muerte violenta o no natural o ésta ha ocurrido en custodia por las autoridades, llevará a cabo un procedimiento que se conoce como inquest (encuesta). La encuesta es un interrogatorio en forma legal de la causa y las circunstancias de la muerte. Es un procedimiento formal efectuado en público por el coroner, que incluye un jurado cuando la muerte ha ocurrido en prisión, bajo custodia policial o por la acción de un policía en cumplimiento de su deber, así como cuando ha ocurrido en el trabajo o se sospecha que pueden producirse más muertes en circunstancias similares. El objeto de la encuesta es exclusivamente el de averiguar la identidad del fallecido y cómo, cuándo y dónde falleció. No tiene acusación y defensa, como en los juicios penales; sólo es una investigación limitada a determinar lo que pasó (inquest is a limited, fact- finding inquiry), y no determina ninguna cuestión sobre la responsabilidad o la culpa.

Normalmente se inicia la encuesta poco tiempo después del fallecimiento para dejar constancia de la muerte, identificar al fallecido y evitar los retrasos en la inhumación o cremación del cadáver; se suspende entonces hasta que las investigaciones policiales o de otros organismos y la propia del coroner se completan. Si hay una persona acusada de la muerte que investiga el coroner, por ejemplo, por asesinato, en la mayoría de los casos se aplaza la encuesta hasta la finalización del correspondiente juicio penal; de aplazarse, el coroner debe enviar al registro los detalles necesarios para inscribir la muerte. El coroner decidirá tras el juicio sobre la reanudación de la encuesta, lo que puede no ser necesario.

Una encuesta suele tardar de media algo menos de siete meses en realizarse, aunque en ocasiones complicadas puede durar más tiempo. El coroner decide quién debe comparecer como testigo en ella; hay obligación de asistir si se vive en Inglaterra y Gales (está penado con una multa el no hacerlo ${ }^{11}$ ). Los testigos son interrogados primero por el coroner. Posteriormente pueden serlo por aquellos legítimamente interesados en el caso, como los familiares directos o sus representantes legales, o representantes de entidades como compañías de seguros, inspectores ministeriales, sindicatos, la propia Policía, etc. Es el coroner el que decide quién está legítimamente interesado en el caso y si las cuestiones que quieren preguntarse son relevantes o no para la encuesta.

Las posibles conclusiones de una encuesta, habitualmente llamadas veredictos, incluyen: causas naturales, accidente, suicidio, unlawful killing (muerte ilícita, la causada por otra persona sin una excusa legítima y con violación de la ley penal, que incluye asesinato, homicidio, infanticidio y la muerte por conducción peligrosa), lawful killing (muerte lícita, el llamado homicidio justificado), enfermedad profesional y veredicto abierto, cuando no hay suficientes pruebas para ningún otro veredicto. Como alternativa, el coroner puede dar un veredicto narrativo que exponga con más detalle los hechos que rodearon a la muerte y explique las razones de la decisión.

\section{Situación actual}

En 2001 había 140 coroners en Inglaterra, de los cuales 25 eran a tiempo completo, todos titulados en leyes, con un coste del servicio de unos 50 millones de libras esterlinas al año ${ }^{12}$. En 2003, la amplia revisión ya mencionada del sistema coroner encontró que las muertes enviadas al coroner eran el 
$38 \%$ del total, mientras que la tasa de autopsias era del $22,8 \%$ de todas las muertes, tasa más del doble que la de cualquier país estudiado, incluidos Irlanda, Canadá, Nueva Zelanda o Australia² ${ }^{2}$ En 2011, con el territorio de Inglaterra y Gales dividido en 113 jurisdicciones, 222.271 muertes fueron comunicadas al coroner, lo que se estima el $46 \%$ del total. De las muertes enviadas al coroner, se autopsiaron el $42 \%$ (lo que representa un 19\% del total de las muertes), porcentaje que confirma una tendencia descendente de los últimos años. El porcentaje de encuestas, iniciadas en el $14 \%$ de las muertes comunicadas al coroner (alrededor del 6\% del total de las muertes) muestra por el contrario una tendencia ligeramente ascendente $^{13}$. No se han encontrado datos publicados sobre la actividad médico legal total en España, por lo que, a modo de comparación, sirvan los datos del Instituto de Medicina Legal en el que trabaja el autor, que abarca a las provincias españolas de Palencia, Salamanca y Valladolid, hechos públicos por el Consejo General del Poder Judicial ${ }^{14}$. A partir del número de fallecimientos de tres provincias (fuente: Instituto Nacional de Estadística ${ }^{15}$ ) en el año 2011 , el porcentaje de investigaciones medicolegales es aproximadamente del 5\%, y el de fallecidos autopsiados por los servicios médico forenses, sobre el $3 \%$ (porcentaje parecido al de Alemania, el $2 \%{ }^{16}$ ).

El sistema coroner en Inglaterra y Gales está actualmente en vías de reforma tras la sanción real el 12 de noviembre de 2009 de la Coroners and Justice $A c t^{17}$. Contiene dos reformas principales: una nueva estructura para el sistema coroner, que será encabezado por un Chief Coroner, y un nuevo sistema de certificación de las defunciones ${ }^{18}$. La nueva legislación introduce la figura del Chief Coroner, con las funciones principales de establecer estándares, asegurar la formación y dar apoyo para promover prácticas homologables en todo el país, así como conocer de los recursos contra las decisiones de los coroners $^{19}$. En el curso de este proceso de reforma del sistema coroner, que se prevé entre en vigor en 2013 , se ha nombrado en mayo de 2012 primer Chief Coroner al magistrado Peter Thornton ${ }^{20}$. En cuanto a los certificados de defunción, todos los ordinarios, los que no se someten al coroner, serán supervisados por una nueva figura dependiente de la atención primaria denominada medical examiner (no confundir con la del mismo nombre que a continuación se describe). Sobre estas reformas, se ha señalado el problema que representará el que la nueva legislación relativa a los coroners sea responsabilidad del departamento de justicia (Ministry of Justice) mientras que el sistema de control de los certificados médicos de defunción por los medical examiners dependa de otro diferente, el de sanidad (Department of Health) ${ }^{5}$.

\section{El sistema medical examiner}

Es un sistema que pone a la cabeza de la investigación de las muertes a un profesional especializado. Característicamente son sistemas dirigidos por patólogos forenses que determinan las causas de la muerte pero generalmente no investigan sus circunstancias (al menos no en un sentido tan amplio como el de los coroners, ya que no realizan encuestas). Normalmente proporcionan servicios forenses a la policía y a los servicios de investigación criminal. Son funcionarios públicos y sus investigaciones son administrativas y no judiciales, pero sus decisiones, como las de cualquier otro funcionario público, pueden ser sometidas a control judicial. Se desarrolló inicialmente en algunas grandes ciudades de los Estados Unidos, si bien como la investigación de las muertes es estatal y no federal y en muchos Estados está delegada a ciudades y condados, coexisten y se mezclan en la actualidad los sistemas coroner y medical examiner ${ }^{2}$. Se diferencia el segundo del primero en que, con pocas excepciones, los coroners son personas legas en Medicina que obtienen su cargo en unas elecciones y que dependen del personal médico a su disposición para la investigación de las muertes y la práctica de las autopsias, mientras que normalmente los medical examiners son médicos y patólogos contratados para el cargo y que a menudo tienen una formación específica en la investigación medicolegal de la muerte y la realización de autopsias forenses ${ }^{21}$. Como ventajas e inconvenientes de uno y otro sistema en América, se ha señalado que los coroners tienen más autonomía, más acceso al poder a través de la estructura política local y más capacidad de representar la voluntad del electorado ya que es un puesto elegido, por tanto con una mayor igualdad con otros cargos similares. Asimismo, cuentan con poderes para citar a las personas y proceder a una encuesta. Sus principales desventajas son la limitación de recursos, ya que son puestos generalmente sostenidos por los presupuestos de los condados, la carencia de conocimientos médicos ya comentada y un posible conflicto de intereses, especialmente si actúan como coroners fiscales, sheriffs o funerarios (estos últimos representan un cuarto de los elegidos). Por otro lado, el sistema medical examiner permite una mayor uniformidad y consistencia en los procesos de investigación de las muertes ya que los requisitos de formación, acreditación y educación continuada son similares para la mayoría de los medical examiners ${ }^{22,23}$.

\section{Historia}

Jentzen ofrece una visión global para los interesados en la historia de la investigación de la muerte en 
América ${ }^{24}$. Los primeros colonos trajeron con ellos a América el sistema coroner tal y como existía en los primeros años del siglo XVII. El primer antecedente de una encuesta realizada por un coroner en los Estados Unidos consta en Nueva Inglaterra en 1635. Al principio, por motivos religiosos no se efectuaban autopsias, con lo que la investigación de la causa de la muerte se limitaba al examen externo y a las declaraciones de los testigos; a partir de 1647 se permitió una cada cuatro años en el cuerpo de un criminal para la enseñanza de la medicina. En 1665 se practicó la primera autopsia médico legal, en un caso de asesinato ${ }^{25}$. El primer reconocimiento oficial de que la implicación de la profesión médica era deseable en las investigaciones de las muertes ocurrió en 1860, cuando se promulgó una ley en el Estado de Maryland que autorizaba al coroner a requerir la participación o asistencia de un médico. En 1868, un médico fue nombrado coroner en una importante ciudad de dicho Estado. En 1877, el Estado de Massachusetts reemplazó sus coroners por médicos, que fueron descritos como medical examiners, por lo que parece el primer uso oficial del término en los Estados Unidos. El primer sistema medical examiner oficial hay que atribuírselo a la Ciudad de Nueva York en $1918^{26}$. Se estableció un sistema médico legal en el que la persona nombrada Chief Medical Examiner tenía que ser un médico experimentado en el campo de la Patología. El sistema describía el tipo de casos que caían bajo la jurisdicción del medical examiner, estipulaba que éste podía efectuar autopsias cuando lo considerara necesario y establecía un laboratorio para su uso. Los casos sometidos al sistema medical examiner eran muertes violentas (accidentes, suicidios, homicidios), muertes sospechosas, muertes súbitas e inesperadas y muertes sin asistencia médica. La mayoría de los sistemas medical examiner en los Estados Unidos son variaciones del concepto original de Nueva York ${ }^{27}$. En conjunto, hubo un desarrollo significativo de los sistemas medical examiner en los 30 años siguientes a 1960, con una disminución en los 90. Desde 2000 a 2007, seis condados han transformado sus sistemas coroner en medical examiner. En este último año, sobre el $31 \%$ de los 3.187 condados de los Estados Unidos están atendidos por medical examiners, 22 Estados tienen un sistema medical examiner sin coroners, de los cuales 19 lo tienen centralizado para todo el Estado, 2 en cada uno de sus condados y 1 agrupado por distritos que abarcan varios condados. Otros 14 Estados tienen sistemas mixtos, con un medical examiner en alguno de sus condados ${ }^{26}$. Como puede verse, los sistemas de investigación de la muerte en Norteamérica presentan tanta diversidad geográfica, incluso dentro de los límites de un mismo Estado, que su heterogénea situación ha sido descrita empleando el símil de una labor de retazos o patchwork. Sobre el $20 \%$ de los 2,4 millones de muertes anuales en los Estados Unidos son investigadas por los coroners o medical examiners, unas 450.000 investigaciones medicolegales al $\mathrm{año}^{23}$.

\section{Funciones}

Para explicar el funcionamiento general del sistema medical examiner seguimos a DiMaio y DiMaio ${ }^{27}$. Son muy críticos con el sistema coroner y llegan a decir que "el sistema coroner se desarrolló en un tiempo en el que el público lego sabía tanto sobre la ciencia de la medicina como los médicos que la practicaban. Los tiempos han cambiado". Afirman que, como regla general, un medical examiner hace un trabajo mucho mejor y más científico que un coroner. Contraponen al argumento para la conservación del sistema coroner de que es más económico que un sistema medical examiner, el que el ahorro se pierde si los casos son invariablemente mal llevados, con el resultado de una litigiosidad cara y prolongada. Sostienen que ninguna oficina medical examiner debería funcionar subordinada a una agencia de policía; en algunos sitios, la oficina medical examiner funciona bajo el Departamento de Salud Pública, lo que puede resultar o no, pero tiende a aumentar la burocracia. Los requisitos básicos de una oficina medical examiner son, primero, una ley adecuada. Bajo tal ley, deberían estar bajo su jurisdicción las muertes violentas (accidentes, suicidios y homicidios), las muertes sospechosas, las muertes súbitas e inesperadas, las muertes sin asistencia médica y las muertes en los calabozos o en instituciones penitenciarias; incluso, en muchas áreas, debe informarse al medical examiner de la muerte de cualquier individuo en las primeras veinticuatro horas de su ingreso hospitalario. La ley, por supuesto, debe establecer que el medical examiner tiene derecho a efectuar una autopsia cuando lo crea necesario. En segundo lugar es preciso un personal cualificado, empezando por el medical examiner jefe, que ha de ser un patólogo forense titulado y con experiencia. Asimismo, son necesarias una plantilla de personal, unas instalaciones y una instrumentación apropiadas. Finalmente, un presupuesto económico que haga todo lo anterior posible.

En cuanto a la organización concreta de las oficinas medical examiner, estos autores prosiguen diciendo que en la mayoría de los sistemas medical examiner de los Estados Unidos la comunicación de las muertes no se hace directamente al patólogo forense, sino a los investigadores no médicos empleados en su oficina, los cuales, tanto si el caso es aceptado como 
si no, redactan un informe detallado que es revisado por el medical examiner tan pronto como sea posible. La razón para no usar médicos en esta tarea es tanto económica como logística. En una comunidad de un millón de personas, puede haber de 4.000 a 6.000 llamadas por muertes al año. Tener un médico que cribe personalmente tales llamadas es caro y una pérdida de tiempo profesional. En lo que se refiere a la carga de trabajo de los medical examiners, si uno efectúa más de 250 autopsias al año se considera un deficiencia denominada Fase I por el programa voluntario de acreditación e inspección instituido en 1997 por la NAME (Nacional Association of Medical Examiners) y si efectúa más de 400 se considera de Fase II: una única deficiencia de este último tipo impide la acreditación. La carga de trabajo para un patólogo forense sin responsabilidades administrativas es de 250 autopsias al año. Por un corto periodo de tiempo se pueden efectuar autopsias a una frecuencia anual de 300, tal vez 325. Desde el momento en que la carga de trabajo excede de 350 autopsias, se cometen errores y se sacrifica su calidad.

\section{Situación actual}

En el año 2009, la National Academy of Science publicó un informe sobre las ciencias forenses en los Estados Unidos ${ }^{28}$, en el que Ilegaron a las conclusiones que se exponen a continuación. En conjunto, los sistemas medical examiner/coroner funcionan con niveles variables de capacitación, a menudo con deficiencias en instalaciones, personal, formación y capacitación, la mayoría de ellos cortos de presupuesto y personal. No hay una obligación a nivel nacional de poseer unos títulos o certificaciones determinados para ejercer de investigadores en este campo. La conversión de los sistemas coroner a medical examiner está detenida y requiere incentivos del gobierno. Hay escasez de patólogos forenses, debido a que resulta poco atractiva esta dedicación de cara a devolver los créditos solicitados por los graduados en medicina para sus estudios, lo que ha llevado a plantear una posible condonación de los mismos a cambio de años de servicio en patología forense. No se utilizan tecnologías diagnósticas actualizadas para investigar la muerte, por ejemplo las modernas pruebas de imagen; el personal no las utiliza por deficiencias presupuestarias o en su formación. La investigación tanto básica como traslacional en patología forense es prácticamente inexistente. Hay incomunicación entre las distintas unidades por la falta de estandarización de las mismas y la multiplicidad de sistemas empleados en la recogida de los datos. El comité que redacta el informe propone la creación de una entidad federal independiente para contribuir a la solución de estos problemas.

\section{La investigación de las muertes innaturales en España}

En España contamos con un sistema civil para la investigación de las muertes innaturales. Frente a un sistema coroner que investiga por igual todas las muertes innaturales, el hecho de que la investigación se centre en el posible delito asociado a la muerte y no en la muerte en sí misma, hace que el investigador oficial, sea juez o fiscal, cese en sus averiguaciones si de ellas no resulta cualquier tipo de ilícito penal. Se afirma que la existencia de encuestas públicas para averiguar las circunstancias de una muerte representa una parte importante de la comprensión del suceso por parte de los seres queridos del fallecido y de la resolución de su duelo, lo que consideran beneficioso profesionales de la investigación de la muerte que no cuentan con este recurso ${ }^{29}$.

Si bien la legislación actual coloca al médico forense a las órdenes directas del Juez de Instrucción, en este momento se prevé que habrá una futura asunción de la labor de investigación de las muertes innaturales por parte del Ministerio Fiscal. La investigación de las muertes por el acusador público no es una situación infrecuente en los sistemas civiles, más bien al contrario. Concretamente en Francia, prototipo de estos sistemas, las muertes violentas, las que ocurren en custodia o por acción de la policía y las muertes derivadas de desastres de masas se comunican al Procureur de la République (fiscal), lo que da lugar automáticamente a una enquête judiciaire (instrucción o investigación judicial). La investigación de las muertes sospechosas también es competencia del fiscal en Italia, Alemania y Países Bajos, entre otros países ${ }^{1}$. Dentro de los países anglosajones, Escocia, aunque influenciada por Inglaterra, mantiene un sistema de investigación de tipo civil, en el cual el Procurator Fiscal, comparable al fiscal de muchos sistemas continentales, tiene amplios poderes en la investigación de materias delictivas ${ }^{30,31}$.

Sin embargo, el paso de una investigación dirigida por el juez a una dirigida por el fiscal puede plantear una serie de problemas. El primero y más destacado de ellos ha de ser el que se deriva de la ausencia en sí misma del Juez en el levantamiento del cadáver, ya que se produce un notable rebajamiento del valor probatorio de cualquier diligencia de inspección ocular. De hecho, esas diligencias de levantamiento sin Juez no podrían tener carácter de prueba preconstituida al 
faltar el requisito subjetivo: no son practicadas ante el Juez de Instrucción ${ }^{32}$. Por otro lado, el médico forense por definición trata siempre con problemas derivados de la salud de los individuos, cuyos datos están especialmente protegidos por el artículo 7 de la Ley Orgánica 15/199933; como las decisiones sobre el uso de tales datos la ha de tomar siempre un juez, una investigación dirigida por éste siempre actuará en este terreno de forma más inmediata y, por tanto, eficaz, que si la dirige un fiscal, quien debe solicitar autorización para el manejo de estos datos protegidos a un juez de garantías.

Ya se planteó la posibilidad en un trabajo anterior ${ }^{34}$ de tomar como modelo la figura del coroner para la ampliación normativa del escueto marco actual de referencia para el médico forense en la investigación de los fallecimientos, puesto que "los casos de muerte en los que interviene el coroner resultan bastante similares a los que obligan a la intervención del Médico Forense en su doble función de Médico del Registro Civil y del Juzgado de Instrucción de guardia, ya que la labor del coroner está limitada exclusivamente al establecimiento de la identidad del fallecido y al de la causa y circunstancias de la muerte. Es asimismo notable el parecido entre estas funciones y las que desempeña el Médico Forense al amparo de lo establecido en el artículo 778.6 LECrim, con la señalada desventaja para éste de que lo hace sin ningún amparo normativo que desarrolle su labor. Por tanto, la existencia de una figura específica y autónoma para investigar las muertes no es rara avis in terris y (...) el Médico Forense tradicional podría asumir la labor que el Juez de Instrucción progresivamente tiende a abandonar". Se propone, en concreto, que la ley manifieste explícitamente la autoridad del médico forense en los levantamientos, los momentos en que debe comunicarse al juez (o al fiscal) los hallazgos que surjan en la diligencia y, de no menor importancia, la posibilidad de acceso a los documentos clínicos necesarios. Asimismo, que se elabore un protocolo de actuación en los casos en los que el médico forense efectúa los levantamientos de cadáver sin juez y, finalmente, que se integren en este tipo de actuaciones las requeridas por el Registro Civil en las muertes sin certificado.

\section{Agradecimientos}

A la Profesora de Derecho Procesal de la Universidad de Valladolid Da Yolanda Palomo Herrero, por la revisión del texto y sus comentarios.

A los compañeros del Instituto de Medicina Legal de Palencia, Salamanca y Valladolid.

Los autores declaran no tener conflicto de intereses.

\section{Bibliografía}

1. Matthews P. Jervis on Coroners. 12th Edition. London: Sweet and Maxwell, 2002.

2. Luce $T$ (Chair). Death Certification and Investigation in England, Wales and Northern Ireland: The Report of a Fundamental Review. London: The Stationery Office, 2003. Disponible en: http:// www. archive2. official-documents.co.uk/document/ cm58/5831/5831.pdf (acceso el 8.11.12).

3. Knight B. The Medieval English Coroner System. Parts 1-6. Páginas web disponibles en: http://www.britannia.com/history/index.html (acceso el 8.11.12).

4. The Coroners' Society of England and Wales. A Brief History. Página web disponible en http://www. coronersociety.org.uk/wfBriefHistory.aspx (acceso el 8.11.2).

5. Palmer R. Death and the coroner. Some reflections on current practice and proposed reforms. Med Sci Law 2012;52:63-70.
6. Cordner SM Loff B. 800 years of coroners: have they a future? The Lancet. 1994;344:799-801.

7. Department of Culture, Media and Sport. The Treasure Act: Information for finders of Trasure (England and Wales). Disponible en: http://www. culture.gov.uk/images/publications/treasurefinders. pdf (acceso el 8.11.12)

8. Ministry of Justice. Guide to Coroners and Inquests and Charter for coroner services. March 2012. Documento en PDF disponible en: http://www. justice.gov.uk/coroners-burial-cremation/coroners (acceso el 8.11.12).

9. Dimond B. The coroner's jurisdiction. 1: the current law in the UK. Br J Nurs. 2004;13:1151-2.

10. Fisher RS. History of Forensic Pathology and related laboratory sciences. En: Spitz WU, Fisher RS. Medicolegal Investigation of Death. 2nd Edition. Spingfield: Charles C Thomas, 1980 
11. Coroners Act 1988. Chapter 13. Disponible en: http:// www. legislation.gov.uk/ukpga/1988/13/contents (acceso el 8.11.12).

12. Samuels A. Coroners. Med Leg J. 2001;69:37-9.

13. Ministry of Justice. Coroners Statistics 2011 England and Wales. Published 17 May 2012. Disponible en: http://www.justice.gov.uk/statistics/coroners-andburials/deaths (acceso el 8.11.12).

14. Instituto de Medicina Legal de Palencia, Salamanca y Valladolid. Memoria anual 2011. Disponible en: http://www.poderjudicial.es/cgpj/es/Poder Judicial/ Tribunales_Superiores_de_Justicia/TSJ_Castilla_y_ Leon/Sala de prensa/Ārchivo de notas de prensa/ Memoria_2011_Instituto_de_Medicina_Legal_Palencia_Salamanca_y_Valladolid (acceso el 8.1̄̄.12).

15. Instituto Nacional de Estadística. Movimiento natural de la población. Datos avanzados: Año 2011. Disponible en: http://www.ine.es/jaxi/menu. do?type $=$ pcaxis \&path $=/$ t20/e301/provi\&file $=$ pcaxis (acceso el 8.11.12).

16. Madea B Rothschild M. The Post Mortem External Examination: Determination of the Cause and Manner of Death. Dtsch Arztebl Int. 2010;107(33):575-88.

17. Coroners and Justice Act 2009. Chapter 25. Disponible en: http://www.legislation.gov.uk/ukpga/2009/25/ contents (acceso el 8.11.12).

18. Luce T. Coroners and death certification law reform: the Coroners and Justice Act 2009 and his aftermath. Med Sci Law. 2010;50:171-8.

19. Samuels A. Deaths and Coroners: Coroners Act 2009. Med Leg J. 2011;79:26-8.

20. The Coroners' Society of England and Wales. The First Chief Coroner of England and Wales is named [22/05/2012]. Página web disponible en: http://www.coronersociety.org.uk/wfPublicAnnDet. aspx?id=97 (acceso el 8.11.12).

21. Hanzlick R Combs D. Medical Examiner and Coroner Systems: History and Trends. JAMA.1998;279:8704.

22. Godwin TA. End of Life: Natural or Unnatural Death Investigation and Certification. Dis Mon. 2005;51:218-77.

23. Committee for the Workshop on the Medicolegal Death Investigation System. Medicolegal Death Investigation System: Workshop Summary. Washington: The National Academies Press 2003. Disponible en: http://www.iom.edu/Reports/2003/MedicolegalDeath-Investigation-System-Workshop-Summary. aspx (acceso el 8.11.12).

24. Jentzen JM. Death Investigation in America. Coroners, Medical Examiners and the Pursuit of Medical
Certainty. Cambrigde, Massachusetts and London, England: Harvard University Press, 2009.

25. Spitz DJ. History and Development of Forensic Medicine and Pathology. En: Spitz WU, Spitz DJ (eds.). Spitz and Fisher's Medicolegal Investigation of Death: Guidelines for the Application of Pathology to Crime Investigation. 4th Edition. Springfield: Charles C Thomas, 2006

26. Hanzlick R. The Conversion of Coroners Systems to Medical Examiner Systems in the United States: A Lull in the Action. Am J Forensic Med Pathol. 2007;28:279-83.

27. DiMaio VJ, DiMaio D. Forensic Pathology. 2nd Edition. Boca Raton: CRC Press, 2001.

28. Committee on Identifying the Needs of the Forensic Sciences Community, National Research Council. Strengthening Forensic Science in the United States: A Path Forward. Washington: The National Academies Press 2009. Disponible en: http://www.nap. edu/catalog.php?record_id $=12589$ \#toc (acceso el 7.12.12)

29. Dean P. The future of the coroner service: a medical examiner system in all but name. J Clin Forensic Med. 2004;11:231-2.

30. University of Dundee, Department of Forensic Medicine, Lecture Notes. Death Investigation: Procurator Fiscal \& Coroner. Disponible en: http://www.dundee. ac.uk/forensicmedicine/notes/notes.html (acceso el 8.11.12).

31. Crown Office and Procurator Fiscal Service. The Role of the Procurator Fiscal in the Investigation of Deaths: Information for Bereaved Relatives. Disponible en: http://www.crownoffice.gov.uk/about/ sudden-suspicious-and-unexplained-deaths (acceso el 8.11.12).

32. Lledó González CL. Inspección ocular, levantamiento de cadáver y recogida de muestras. Manuales de Formación Continuada, Serie Penal, n 46/2007. Madrid: Consejo General del Poder Judicia,I 2007.

33. Ley Orgánica 15/1999, de 13 de diciembre, de Protección de Datos de Carácter Personal. Boletín Oficial del Estado. 1999;298:43088-99.

34. Sánchez Pérez JD Palomo Herrero Y. La actuación del Médico Forense en virtud del artículo 778.6 de la Ley de Enjuiciamiento Criminal: "Si ves algo raro, me llamas". Boletín del Ministerio de Justicia. 2010;2120:6-27. Disponible en: http://www.mjusticia.gob.es/cs/Satellite/1292342419327?blobheade $\mathrm{r}=$ application/pdf\&blobheadername1 = Content-Disp osition\&blobheadername $2=$ Boletin\&blobheadervalu e1 $=$ attachment; + filename $=$ Estudios. PDF\&blobhea dervalue $2=1288774337170$ (acceso el 8.11.12) 